\title{
Comparaison de dosimètres individuels de neutrons pour utilisation autour du futur réacteur TRIGA du Maroc : détecteur à bulles et détecteur solide de traces nucléaires de type CR-39
}

\author{
M. EL MESSAOUDI ${ }^{1}$, A. CHOUAK ${ }^{\prime}$, M. LFERDE ${ }^{1}$
}

RÉSUMÉ I a dosimétrie individuelle présente plusieurs difficultés dues aux différences des réponses en énergie des dosimètres. Dans ce travail, nous proposons un dosimètre individuel réglementaire pour la surveillance radiologique des personnes soumises à l'exposition neutronique, autour du futur réacteur de recherche de type TRIGA Mark II du Maroc. L'exposition aux neutrons n'aura lieu qu'épisodiquement et les doses reçues devraient rester faibles. Afin de choisir convenablement un dosimètre individuel de neutrons, nous avons étudié deux systèmes : le détecteur à bulles et le détecteur solide de traces nucléaires $\mathrm{PAI}) \mathrm{C}^{2}\left(\mathrm{CR}-39^{\mathrm{K}}\right)$. Vu les limitations imputées aux propriétés dosimétriques des différents dosimètres actuellement employés, une étude comparative approfondie est nécessaire en vue de disposer d'un dosimètre individuel convenable. Cette étude s'inscrit dans le contexte marocain : absence d'installations pour réaliser des tests, un nombre restreint de travailleurs opérant dans des zones spécifiques pourrait être soumis à l'exposition neutronique, contrairiées économiques, champ neutronique souvent mixte et la réglementation qui impose le port d'un dosimètre individuel dans une zone contrôlée. Fn raison de ses caractéristiques dosimétriques et de son prix, le dosimètre de type CR-39 peut être une bonne solution comme dosimètre individuel passif de neutrons autour du réacteur TRIGA. Le dosimètre à bulles, permettant d'instaurer la démarche ALARA, serait utilisé comme dosimètre individuel complémentaire bien adapté à la dosimétrie opérationnelle.

ABSTRACT A comparison of neutron personal dosimeters for use in the future Moroccan TRIGA reactor: bubble detectors and CR-39.

Individual neutron monitoring presents several difficulties due to the differences in energy response of the dosimeters. In this work, we propose a neutron dosimeter for routine surveillance of the occupationally exposed persons around the future TRIGA Mark II reactor in Morocco. The neutron doses will be received infrequently and should remain low. In order to choose the most appropriate neutron personal dosimeter, we have studied two systems: the bubble detectors and the PADC ${ }^{2}$ $\left(C R-39^{(K)}\right)$ solid state nuclear track detector. In view of well-known limitations related to the dosimetric properties of the actual dosimeters used in routine

\footnotetext{
${ }^{1}$ I aboratoire de Physique Nucléaire. Département de Physique. Ficulté des Sciences, avenue Ibn Baltouta. B.P. I0I t, Rabat, Maroc:

Poly allyl diglycal carhonate.
} 
surveillance, a thorough comparative study is necessary to dispose of a convenient dosimeter. This study aims in Morocean context: no facility for realising the tests, few workers operating in specific zones might be exposed to neutrons, economical constraints, mixed field and regulatory system which recommends the use of personal dosimeter in controlled areas. Due to its dosimetric characteristics and its cost, the PADC detector can be a good solution as a passive neutron personal dosimeter for individual monitoring at the TRIGA reactor. The bubble dosimeter, which allows the ALARA principle to be applied, can be used like a complementary dosimeter for operational dosimetry.

\section{Introduction}

La surveillance individuelle fait partie intégrante du dispositif de radioprotection des travailleurs exposés. Son objectif fondamental est d'obtenir une estimation de la dose équivalente et de la dose efficace ; cette information est utile pour limiter les doses reçues par les travailleurs à un niveau de risque acceptable et démontrer le respect des limites réglementaires (Wernli et al., 1996).

Pour la dosimétrie individuelle (neutrons, protons, photons, électrons, ...), la législation nationale impose l'utilisation d'un dosimètre individuel réglementaire dans les zones contrôlées et des mesures avec des rem-mètres dans les zones surveillées (JO, 1997).

Les limites des doses réglementaires utilisées actuellement pour l'exposition des travailleurs et du public sont celles données dans la publication 60 de la CIPR (ICRP, 1991). La dosimétrie opérationnelle n'est obligatoire que dans les zones contrôlées.

Nous ne disposons actuellement pas d'installations pour effectuer des tests pour les neutrons. Dans le futur, la nouvelle structure de radioprotection et de sûreté nucléaire devrait disposer d'une installation pour réaliser des étalonnages et des tests des dosimètres et moniteurs de neutrons, avant leur introduction en usage de routine. Ce travail constitue une aide à la décision du choix du dosimètre individuel passif et dresse un bilan des problématiques que posent la dosimétrie des neutrons.

Pour le moment, le choix d'un dosimètre se fonde sur ses propriétés dosimétriques par rapport aux autres systèmes existants et son retour d'expérience dans des pays ayant largement exploité ce type de dosimètre en usage réglementaire.

Des différents systèmes complets constitués de détecteurs solides de traces, système de développement des traces et lecteur, sont aujourd'hui commercialisés. 
Les difficultés rencontrées en dosimétrie individuelle des neutrons sont principalement dues à l'étendue de la gamme d'énergies du spectre de neutrons et à là grande variété des spectres rencontrés dans les zones de travail, en combinaison avec les facteurs de qualité et les nouveaux facteurs de pondération des neutrons (ICRP, 1991). Jusqu'à présent, il n'y a aucun dosimètre dont la réponse en équivalent de dose couvre la totalité de la plage en énergie des neutrons rencontrée dans la pratique. Ainsi, du fait que tous les dosimètres ont une réponse qui dépend plus ou moins fortement de l'énergie, ils ne fournissent une estimation correcte de la dose que dans des champs de rayonnements proches de celui utilisé pour leur étalonnage. Des méthodes de spectrométrie des neutrons sont employées pour déterminer un facteur de correction local permettant d'ajuster la lecture du dosimètre.

En tenant compte des aspects spécifiques au contexte marocain et des problèmes sus-mentionnés, il est d'intérêt primordial de choisir un dosimètre individuel approprié à la surveillance radiologique des personnes autour du futur réacteur TRIGA. Dans ce travail, nous donnons un aperçu succinct de l'ensemble des dosimètres individuels de neutrons actuellement disponibles. Nous décrirons plus en détail les caractéristiques dosimétriques des deux dosimètres proposés, telles que la dépendance énergétique, la dépendance angulaire et d'autres caractéristiques.

\section{Dosimètres individuels de neutrons les plus courants}

La compilation des spectres de neutrons calculés et mesurés, rencontrés en radioprotection, montre que les spectres de transmission, en particulier dans le cas de protections métalliques, contiennent une composante importante entre 0,1 et $0,5 \mathrm{MeV}$. Néanmoins, il est évident que des neutrons d'énergies inférieures à 0,1 MeV sont également présents autour des réacteurs (IAEA, 1990, 2002). La figure 1 présente deux exemples de spectres typiques de neutrons, mesurés à l'aide des détecteurs à activation, dans deux réacteurs de recherche de types TRIGA et BMRR, à l'air libre à proximité d'un faisceau de neutrons épithermiques utilisés pour des traitements médicaux (BNCT ${ }^{3}$ ) (Serén et al., 1999). On voit clairement que des neutrons rapides sont présents et représentent $20 \%$ dans les deux réacteurs de recherche. On peut conclure que des spectres de neutrons dégradés sont fortement présents dans les lieux de travail, mais également des neutrons rapides en proportion modeste. Des spectres de neutrons calculés dans les lieux de travail d'un réacteur de recherche montrent que les neutrons ralentis (thermiques) sont dominants, mais dans le hall des expériences il y a également des neutrons rapides (Kamenopoulou et al., 2001).

\footnotetext{
Boron neutron capture therapy.
} 


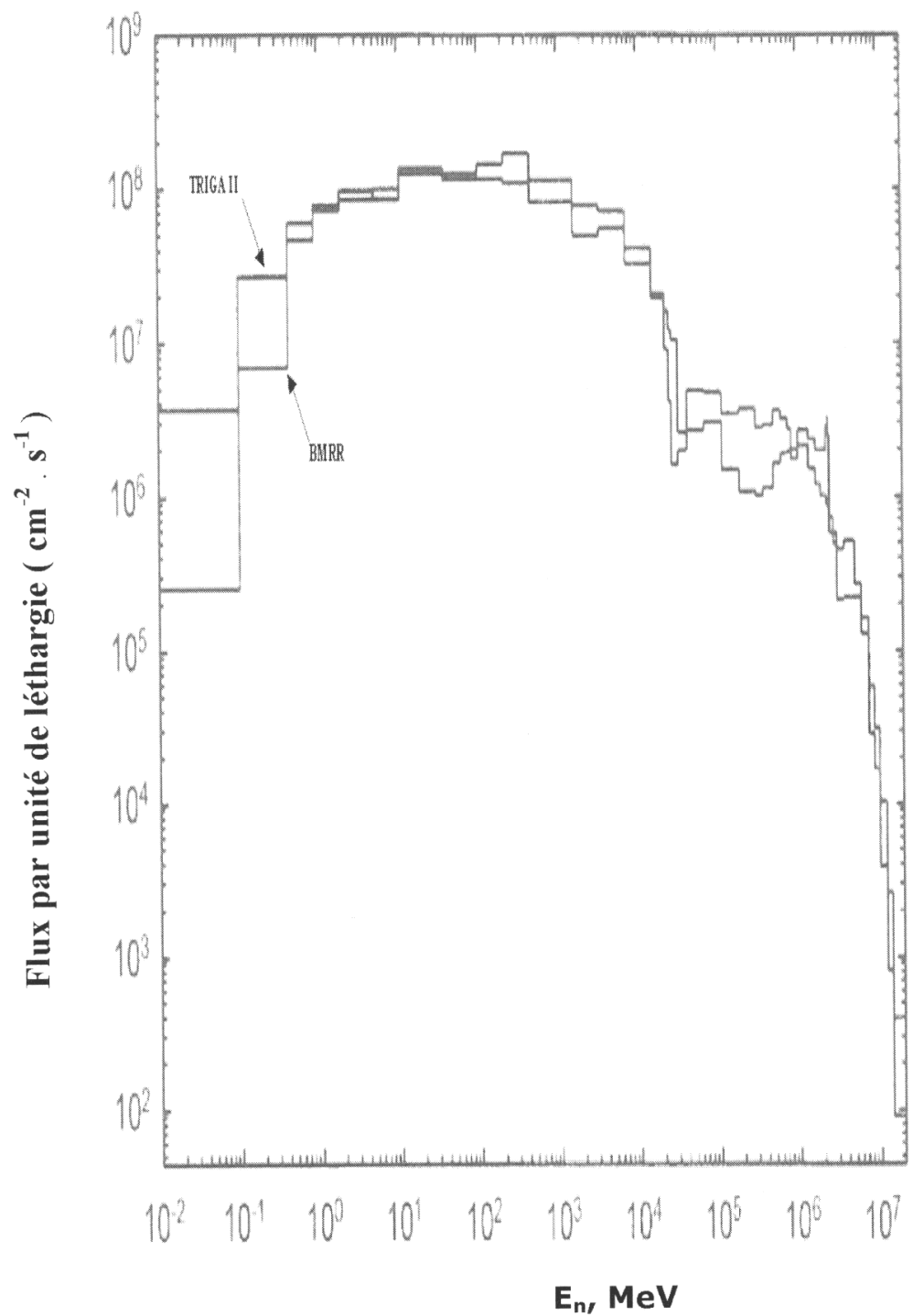

Figure 1 -Exemple de spectres de neutrons à l'air libre au voisinage de BNCT rencontrés dans des réacteurs de recherche de types TRIGA et BMRR; flux par unité de léthargie (Serén et al., 1999).

Typical neutron spectra encountered at free-air around BNCT at TRIGA and BMRR research reactors, in lethargy representation (Serén et al., 1999). 
Le futur réacteur est destiné à des expériences d'analyse par activation avec neutrons ralentis, de radiographie par neutrons, de production de radio-isotopes, BNCT, ... Ces applications exploitent des neutrons ralentis mais des neutrons rapides pourraient être présents dans les lieux de travail.

Les neutrons rapides pourraient également contribuer à l'exposition lors de missions de contrôle de sûreté du réacteur ou de maintenance. Même avec un nombre de neutrons rapides modeste, la contribution de ces neutrons pourrait représenter plus de $50 \%$ de la dose équivalente, même en présence d'une composante importante de neutrons thermiques, en raison des facteurs de conversion (fluence-dose équivalente) très élevés dans la gamme des neutrons (100 keV-1 à $2 \mathrm{MeV}$ ). C'est également dans cette gamme d'énergies que l'équivalent de dose dans les installations nucléaires est le plus important (même en présence de neutrons thermiques). Dans la situation d'exposition envisageable autour du futur réacteur TRIGA, les spectres en énergie seraient extrêmement variés selon les lieux et les protections mises en œuvre. Ils comprennent des neutrons thermiques jusqu'à $1 \mathrm{MeV}$, avec une dominance de neutrons ralentis. Les débits de dose équivalente varient en situation normale de l'ordre de $0,1 \mu \mathrm{Sv} / \mathrm{h}$ (bruit de fond) à quelques dizaines de $\mu \mathrm{Sv} / \mathrm{h}$. Dans certaines situations particulières, les valeurs des débits de dose équivalente pourraient être élevées.

Des systèmes de détection couvrant toute la gamme d'énergies sont indispensables. Notre choix du dosimètre individuel de type CR-39, associé aux convertisseurs, et du dosimètre à bulles, BDT et BD-PND, est justifié par l'étendue de la gamme d'énergies des neutrons que pourraient couvrir ces deux types de dosimètres proposés ici.

Les techniques de dosimétrie individuelle passive les plus couramment employées se fondent sur: les émulsions photographiques nucléaires de type NTA, les détecteurs thermoluminescents à albédo (TLD à albédo), les détecteurs solides de traces nucléaires de type CR-39 et les détecteurs à bulles. Les dosimètres électroniques arrivent sur le marché mais n'ont pas encore donné complète satisfaction jusqu'à présent pour être considérés comme dosimètres réglementaires (Vareille et al., 1997). Pour une dosimétrie opérationnelle, par contre, des dosimètres électroniques sont mis au point et commercialisés (Lahaye et al., 2001). Les caractéristiques typiques de ces dosimètres individuels passifs de neutrons sont présentées dans le tableau I. Aucun d'eux ne peut être considéré comme complètement satisfaisant; tous présentent quelques limitations liées à leurs propriétés dosimétriques : faible réponse aux neutrons rapides (TLD à albédo), réponse angulaire médiocre (CR-39), seuil de détection élevé (NTA, CR39) et seuil d'énergie élevé (NTA). Généralement, l'association de plusieurs types de dosimètres est nécessaire pour couvrir la totalité de la plage en énergie des 


\section{TABLEAU I}

Caractéristiques typiques des dosimètres individuels passifs actuellement en service. La limite inférieure de détection (LID) et la limite supérieure de détection (LSD) sont des valeurs approximatives, qui donnent une indication sur la gamme de dose couverte en pratique par ces différentes techniques (Griffith, 1992).

Typical characteristics of passive neutron dosemeters for individual monitoring introduced in routine service. LLD-lower limit of detection; ULD-upper limit of detection (approximate value indicating practical dose range) (Griffith, 1992).

\begin{tabular}{|c|c|c|c|c|}
\hline 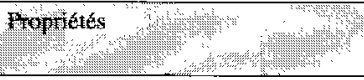 & TIINATA & Albedo (TUD) & CR-39 (DST) & $\begin{array}{l}\text { defecteurs } \\
\text { abulles }\end{array}$ \\
\hline $\begin{array}{l}\text { réponse aux neutrons } \\
\text { rapides }\end{array}$ & $\begin{array}{l}\text { acceptable } \\
>500 \mathrm{keV}\end{array}$ & $\begin{array}{l}\text { faible } \\
>10 \mathrm{keV}\end{array}$ & $\begin{array}{l}\text { acceptable } \\
>100 \mathrm{keV}\end{array}$ & $\begin{array}{l}\text { acceptable } \\
>100 \mathrm{keV}\end{array}$ \\
\hline $\begin{array}{l}\text { réponse aux neutrons } \\
\text { therniques }\end{array}$ & exeellente & élevéc & faible & $\begin{array}{l}\text { excellente } \\
\text { avee BDT }\end{array}$ \\
\hline coût détecteur & faible & modéré & faible & Elevé \\
\hline $\begin{array}{l}\text { sensibuilité } \\
\text { à l'environnement }\end{array}$ & oui & Caible & faible & $\begin{array}{l}\text { température } \\
\text { ef choces }\end{array}$ \\
\hline $\operatorname{LSD}(\mathrm{mSv})$ & $>100$ & $>100$ & $>50$ & $1-10$ \\
\hline $\mathrm{LID}(\mathrm{mSv})$ & $0,3-0,8$ & $0,005-0,2$ & $0,02-0,3$ & $0,005-0,02$ \\
\hline réponse aux photons* & non négligeable & compensable & non & non \\
\hline
\end{tabular}

*En utilisant un système multi-éléments $\left({ }^{6} \mathrm{LiF}-{ }^{7} \mathrm{LiF}\right)$.

neutrons. Bien que n'étant pas parfaits, ils sont couramment utilisés et certains d'entre eux sont commercialisés.

Le dosimètre photographique nucléaire à émulsion NTA détecte les protons de recul, issus des interactions des neutrons avec l'hydrogène, contenu dans l'émulsion et le matériau plastique environnant. L'émulsion nucléaire présente un seuil de détection en énergie de 0,5 à $1 \mathrm{MeV}$ dans le domaine des neutrons rapides et elle est également sensible aux neutrons thermiques. De tels dosimètres ne sont pas très utiles autour des réacteurs, où la contribution à la dose équivalente, de l'ordre de $2 \%$ à $60 \%$, est due aux neutrons ralentis d'énergie intermédiaire, des thermiques à $0,5 \mathrm{eV}$ (Alberts et al., 1996).

Le dosimètre à albédo fondé sur la thermoluminescence, utilisant la différence de lecture entre un TLD de ${ }^{6} \mathrm{LiF}$ et un autre de ${ }^{7} \mathrm{LiF}$, est souvent utilisé en dosimétrie des neutrons. La réponse des dosimètres à albédo est en général fortement dépendante de l'énergie et ne peut être correctement corrigée par la géométrie du dosimètre (Al-Jarallah, 1989). Sa réponse relative en dose équivalente est supérieure à 1 et presque constante pour les neutrons thermiques et épithermiques. Elle décroît rapidement au-delà de $10 \mathrm{keV}$, atteignant une valeur relative d'environ $1 \%$ pour les neutrons rapides (Piesch, 1982). Sa sensibilité en dose équivalente dépend fortement du spectre avec lequel il est utilisé, ce qui 
oblige à appliquer un facteur d'étalonnage propre à chaque poste de travail. Les dosimètres à albédo sont aussi sensibles aux photons et leur réponse angulaire est médiocre. Cette contribution peut être soustraite par le détecteur TL qui est sensible seulement aux photons (dans ce cas TLD avec ${ }^{7} \mathrm{Li}$ ). La limite minimale de détection en terme de dose est comparable à celle des dosimètres thermoluminescents sensibles aux photons ; elle se situe autour de $0,02 \mathrm{mSv}$ dans des champs de neutrons ralentis (Alberts et al., 1996). En raison de son utilisation dans une gamme d'énergies limitée et la nécessité de faire un étalonnage à chaque poste de travail, le dosimètre à albédo fondé sur la thermoluminescence, malgré sa sensibilité élevée aux neutrons ralentis, est peu approprié à nos besoins pour la dosimétrie individuelle des neutrons ralentis autour du réacteur TRIGA.

Les détecteurs solides de traces se sont révélés intéressants depuis que l'on a constaté en 1978 que le détecteur plastique PADC, de nom commercial CR-39, peut détecter les protons d'énergie inférieure à $50 \mathrm{keV}$ et est insensible aux photons (Harvey et $a l$., 1997). Leur principe se base sur la révélation des traces latentes créées dans le détecteur le long des trajectoires des particules de recul chargées (protons, alpha, ions lourds), produites par interaction des neutrons avec le détecteur lui-même ou avec le radiateur. Le nombre de traces obtenues après attaque chimique, électrochimique, ou combinaison des deux, est proportionnel à la dose neutronique dans le détecteur (Hadlock et Parkhurst, 1986). Ce type de détecteur permet la détection des neutrons au-dessus de $50 \mathrm{keV}$ environ, grâce aux réactions ( $n, p)$ ayant lieu dans le détecteur CR-39 ou dans le rayonnateur hydrogéné. Le traitement électrochimique des détecteurs solides de traces de type CR-39 permet d'obtenir un seuil de détection en énergie de l'ordre de $100 \mathrm{keV}$, comparé à $200 \mathrm{keV}$ avec un développement chimique (Harvey et al., 1998). La sensibilité aux neutrons thermiques et épithermiques peut être établie grâce à des convertisseurs comme le bore, le lithium ou l'azote.

Les détecteurs à bulles (BD) décrits par Apfel en 1979 puis les détecteurs à gouttes surchauffées (SDD), sont encore considérés comme relativement nouveaux dans le domaine de la dosimétrie de neutrons. Ils peuvent apporter une réponse pertinente aux besoins de la dosimétrie opérationnelle (d'Errico et al., 1997). Le dosimètre à bulles de type BD-PND est fondé sur un gel porté à l'état métastable. L'énergie libérée dans le gel par des particules à transfert linéique d'énergie (TLE) élevé, lors des interactions des neutrons, provoque une vaporisation des micro-gouttelettes, déjà présentes dans le gel, jusqu'à une taille où elles deviennent visibles et peuvent facilement être comptées à l'œil ou par lecteur automatique. Le nombre de bulles est proportionnel à la dose équivalente dans le dosimètre. Le dosimètre à bulles de type BDT est introduit pour tenir compte de la composante thermique. Quand la dose imputée aux neutrons thermiques est importante, il est nécessaire d'utiliser les deux dosimètres à bulles, 
BD-PND et BDT (Vanhavere et al., 1998). Avec cette association de deux dosimètres à bulles, les critères ISO seraient remplis sur une gamme d'énergie allant de $100 \mathrm{keV}$ à plusieurs $\mathrm{MeV}$, ainsi que pour les neutrons thermiques. Quand la grande partie de la dose est due aux neutrons thermiques en présence d'un fantôme, une sur-estimation de la réponse a lieu aux grands angles (Vanhavere et d'Ericco, 2002).

En présence de spectres inconnus, les dosimètres à bulles et les détecteurs solides de traces de type CR-39 sont les plus appropriés. Dans la suite nous considérons les dosimètres à bulles fabriqués par BTI (Bubble Technology Industries, Chalk River, Canada) et les dosimètres de type CR-39, utilisés par le NRPB (National Radiation Protection Board) comme dosimètres individuels réglementaires pour les neutrons au Royaume-Uni. Nous comparerons plus en détail leurs caractéristiques.

\section{Discussion}

\subsection{Dépendances énergétique et angulaire}

On remarque une différence en terme de réponse en dose équivalente en fonction de l'énergie des neutrons pour une incidence normale, normalisée au ${ }^{252} \mathrm{Cf}$, des deux systèmes proposés. La réponse en dose équivalente est plus importante dans le cas du dosimètre à bulles que dans le cas du détecteur solide de traces de type CR-39.

Un exemple typique de la dépendance énergétique de la réponse en dose équivalente des dosimètres à bulles de type BD-PND est porté sur la figure 2 (Vanhavere et al., 1998). En ce qui concerne les détecteurs solides de traces de type CR-39, les résultats des dosimètres individuels des neutrons obtenus après une combinaison d'attaques chimique et électrochimique à haute fréquence ( $2 \mathrm{kHz}$ ) (Tanner et al., 2002), considérées comme conditions de développement du dosimètre CR-39 recommandées par le NRPD, sont également présentés sur la figure 2. Les incertitudes totales (statistiques et systématiques) sur les réponses en équivalent de dose individuel sont estimées entre de 15 et $20 \%$ et entre 4 et $12 \%$ pour le détecteur CR-39 et le détecteur à bulles BD-PND, respectivement.

Le dosimètre à bulles de type BD-PND montre une réponse en dose équivalente correcte et plutôt plate entre 1 et environ $10 \mathrm{MeV}$. Pour des neutrons d'énergies supérieures à $10 \mathrm{MeV}$, on observe une diminution.

La réponse en dose équivalente du dosimètre de type CR-39 dépend de l'énergie des neutrons et atteint une valeur maximale à $1 \mathrm{MeV}$, comme on peut le 


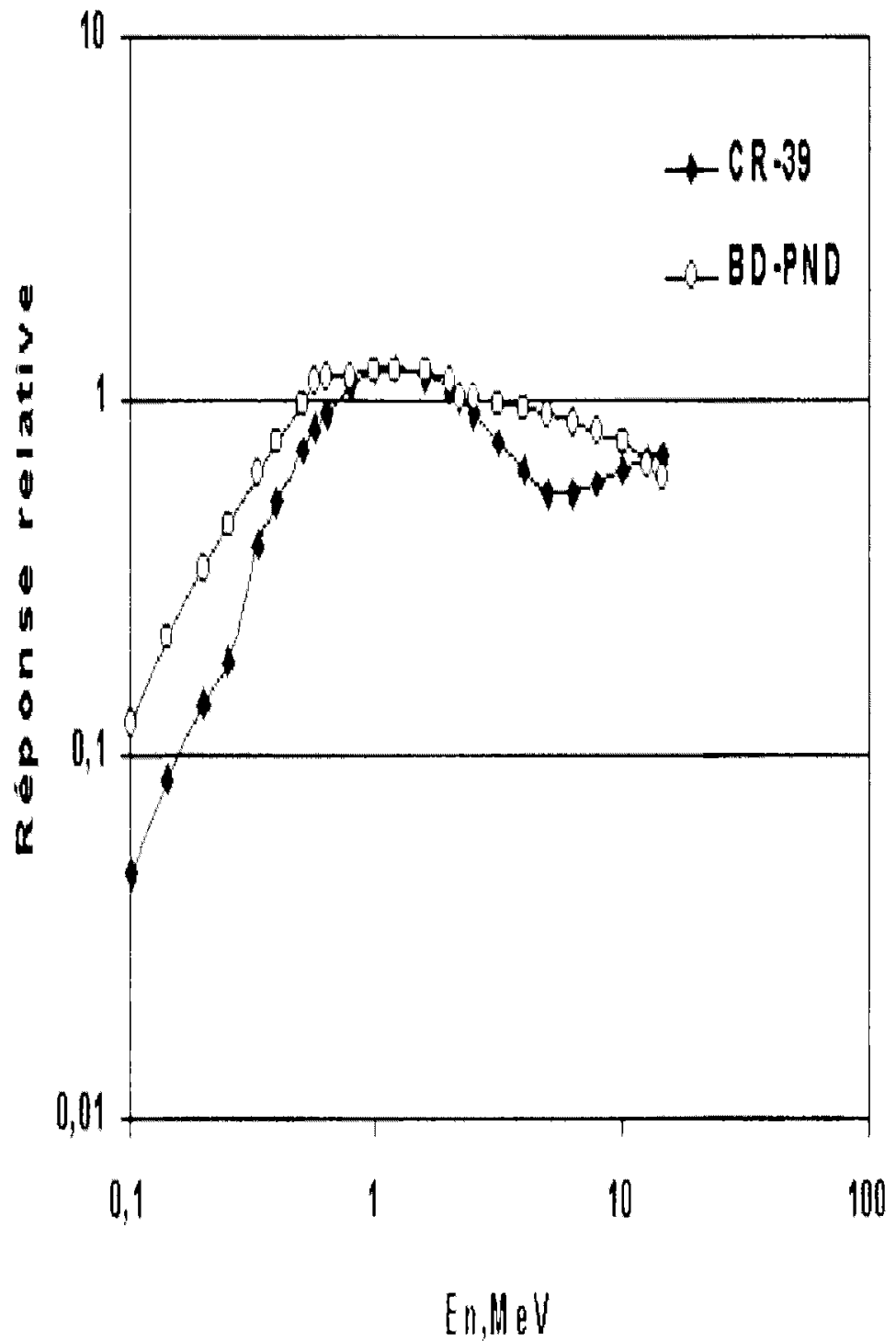

Figure 2 - Dépendance énergétique (relative à ${ }^{252} \mathrm{Cf}$ ) de la réponse en équivalent de dose individuel, en incidence normale, des dosimètres individuels de types CR-39 (Tanner et al., 2002) et BD-PND (Vanhavere et al., 1998).

Energy dependence of personal dose equivalent response (relatively to ${ }^{252}$ Cf) of personal dosemeters CR-39 (Tanner et al., 2002) and BD-PND (Vanhavere et al., 1998), al normal incidence. 
constater sur la figure 2. Cette dépendance n'est pas trop marquée et liée au processus de détection dans le CR-39.

En utilisant le dosimètre de type CR-39, la dépendance énergétique de la réponse est affectée par plusieurs facteurs : (i) la qualité de CR-39, (ii) le type et l'épaisseur du convertisseur, (iii) les paramètres de développement chimique et électrochimique et (iv) les procédures de comptage.

Vue leur forme cylindrique, la réponse des dosimètres à bulles ne change pas trop avec l'angle d'irradiation dans la géométrie air-libre. Ce n'est pas le cas lorsqu'un fantôme est utilisé. Des travaux récents montrent que la réponse angulaire mesurée des dosimètres à bulles de type BD-PND, en utilisant une source de californium $\left({ }^{252} \mathrm{Cf}\right)$, est en bon accord avec les critères ISO.

Cet accord a été aussi observé dans le cas des dosimètres individuels avec des neutrons thermiques et épithermiques de type BDT, sauf aux grands angles.

Pour le détecteur solide de traces de type CR-39, nous présentons les résultats normalisés à une incidence normale (A-P) pour deux géométries d'irradiations (ISO et ROT), sur la figure 3 (Tanner et al., 2002). On remarque une décroissance de la sensibilité quand on change la géométrie d'irradiation en passant de A-P à ROT et de ROT à ISO, aussi bien pour les faibles que pour les grandes énergies. Les incertitudes associées sont celles données dans le paragraphe précédent. La dépendance angulaire peut être corrigée par l'introduction des facteurs de correction locaux, fondés sur la détermination des caractéristiques énergétiques et angulaires des neutrons à l'endroit où le dosimètre est utilisé. Parfois, la détermination de ces facteurs n'est pas une tâche simple et, dans certaines situations, il est plus approprié d'utiliser un dosimètre dont la réponse angulaire a été améliorée en utilisant plusieurs éléments ou en modifiant sa géométrie (Harvey et al., 1998). La dépendance angulaire chute avec l'augmentation de l'angle d'incidence, même en géométrie d’irradiation air-libre (Turek et al., 1995).

\subsection{Linéarité et limite minimale de détection}

Les dosimètres à bulles de type BD-PND sont linéaires jusqu'au point où les bulles commencent à se superposer et la lecture peut ne pas être faite correctement. Selon la méthode de lecture (à l'œil : environ 80 bulles ; avec un appareil spécifique pour les grands nombres de bulles : environ 350 bulles) et la sensibilité, la gamme dynamique de mesure peut être limitée. Les dosimètres à bulles peuvent être choisis selon les doses estimées pour être très sensibles (quelques bulles par $\mu \mathrm{Sv}$ ), donnant une précision élevée et une meilleure limite de détection, ou moins sensibles offrant une gamme dynamique plus élevée mais une précision médiocre. Ce choix doit être fait en fonction des doses prévues. 


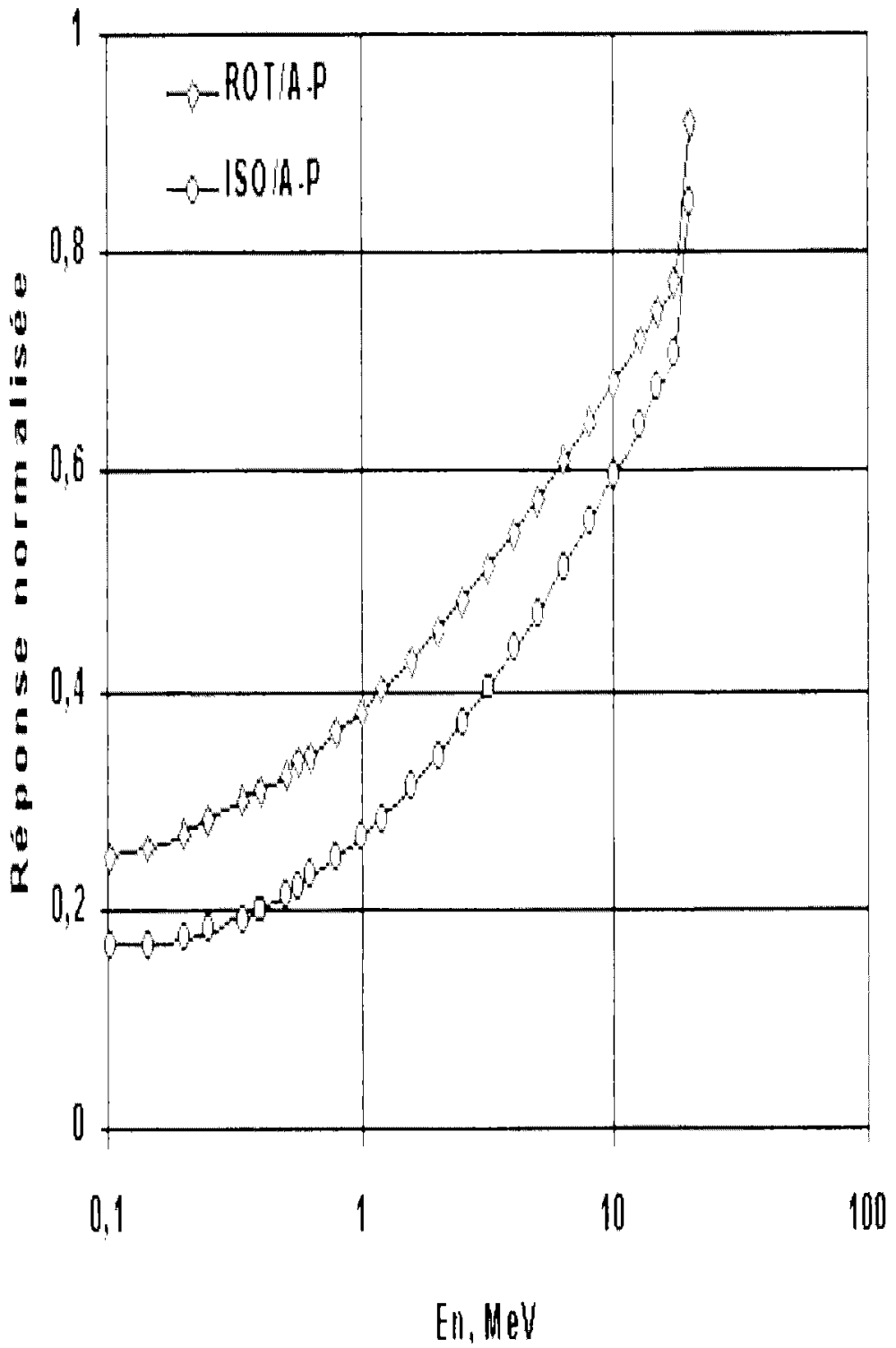

Figure 3-Réponse angulaire aux géométries d'irradiation rotationnelle et isotrope du dosimètre individuel de type CR-39, normalisée à une géométrie A-P (Tanner et al., 2002).

Angular response at rotational and isotropic irradiation geometries of CR-39 dosemeter, normalised to A-P geometry (Tanner et al., 2002). 
Le dosimètre de type CR-39 est linéaire sur l'importante gamme de dose de 0,5 à $5 \mathrm{mSv}$, comme il a été constaté pour les neutrons de $5,3 \mathrm{MeV}$ durant l'exercice EURADOS/CENDOS-1990. Aux doses élevées, la densité des traces observée expérimentalement cesse d'être proportionnelle à l'équivalent de dose auquel le dosimètre est exposé (Harvey et al., 1998). Ce manque de linéarité est rarement un problème, puisque la réponse du dosimètre est connue avec précision sur la gamme entière de doses, entre les faibles doses opérationnelles et celles relativement élevées utilisées dans le but d'étalonnage de routine.

La sensibilité du dosimètre CR-39 dépend des caractéristiques de chaque type de matière plastique CR-39 employée, du type et de l'épaisseur du convertisseur, des paramètres de développements chimique et électrochimique et des procédures de comptage.

La limite minimale de détection par rapport à $\mathrm{H}_{\mathrm{p}}(10)$ obtenue par le NRPB, qui commercialise un dosimètre individuel de type CR-39 complet, est comprise entre 0,02 et $0,10 \mathrm{mSv}$ mais peut être réduite à moins de $0,01 \mathrm{mSv}$ (Harvey et al., 1998).

\subsection{Effet de température}

Le seuil de détection et la sensibilité des détecteurs à bulles dépendent de la température puisque le degré d'ébullition d'un liquide comprimé est fonction de la température. Les détecteurs à bulles de types BD-PND et BDT sont compensés en température, ce qui signifie que la sensibilité devrait demeurer à $\pm 20 \%$ entre 20 et $37^{\circ} \mathrm{C}$. Les critères ISO sont presque satisfaisants entre 20 et $37^{\circ} \mathrm{C}$ pour les neutrons rapides $\left({ }^{252} \mathrm{Cf}\right)$ mais pas pour les neutrons thermiques. Le processus de vieillissement des dosimètres affecte le système de compensation en température ; il en résulte une augmentation élevée de la sensibilité aux températures élevées. Il est aussi important de noter qu'avec l'augmentation de la température, il y a également une diminution du seuil d'énergie et une augmentation de la sensibilité (Vanhavere et al., 1999).

Pour ce qui concerne le détecteur solide de traces de type CR-39, les changements ne sont généralement pas grands à la température ambiante mais ils deviennent significatifs au voisinage de $40^{\circ} \mathrm{C}$ (Harvey et al., 1997). En général, tous les changements qui pourraient être reproduits durant des périodes normales d'utilisation peuvent facilement être corrigés. L'irradiation par des particules alpha entraînait une variation de la réponse de moins de $15 \%$, dans la gamme normale de la température rencontrée $\left(10\right.$ à $\left.40^{\circ} \mathrm{C}\right)$, par rapport à la réponse à la température de $22^{\circ} \mathrm{C}$ (Bartlett, 1987). 


\section{Conclusion}

Deux techniques pour la dosimétrie individuelle des neutrons sont présentées dans la perspective de la mìse en service du futur réacteur TRIGA du Maroc.

Étant donné que les neutrons sont pratiquement toujours accompagnés de rayons gamma, il est indispensable de disposer d'une dosimétrie séparée pour chacun de ces rayonnements. Les deux dosimètres proposés satisfont parfaitement ce critère d'évaluation des doses neutroniques en champ mixte, en raison de leur insensibilité aux photons gamma.

Concernant les dosimètres à bulles, leurs bonnes performances dosimétriques sont claires : sensibilité élevée, réponse normalisée en dose équivalente plutôt plate sur la plage de $100 \mathrm{keV}$ à $1 \mathrm{MeV}$ entre $-80 \%$ et $+10 \%$, réponse angulaire acceptable, insensibilité aux photons gamma et plus basse limite de détection. L'association des dosimètres à bulles de types BDT et PND permettrait de couvrir la gamme d'énergie très étendue allant du thermique à quelques $\mathrm{MeV}$.

Le travail dans une zone contrôlée aux niveaux élevés de dose montre la nécessité de la mise en ceuvre de mesures en temps réel quand un certain niveau de dose équivalente est dépassé. Les dosimètres à bulles pourront remplir cette condition. En raison de leur lecture directe, les dosimètres à bulles permettent de mettre en place le principe ALARA comme pour les dosimètres électroniques des photons. Ils seront donc très utiles comme dosimètres actifs complémentaires, pendant des périodes limitées et pour des applications spéciales, malgré leur prix élevé.

L'intérêt principal des dosimètres de type CR-39 est leur gamme d'énergies étendue, du thermique à plusieurs $\mathrm{MeV}$, comparés aux dosimètres thermoluminescents à albédo et à émulsion nucléaire. L'étendue de la gamme d'énergie est particulièrement intéressante pour couvrir l'ensemble des situations autour des réacteurs, où une contribution significative à la dose équivalente est due aux neutrons d'énergies inférieures à $1 \mathrm{MeV}$. Les spectres de neutrons rencontrés dans le futur réacteur TRIGA comprennent des neutrons thermiques, intermédiaires et rapides, avec des flux relatifs dépendant du lieu d'exposition et une dominance des neutrons ralentis. Ainsi, le travailleur qui se déplace souvent durant son activité est exposé, de façon aléatoire, à un rayonnement incident variable en spectre et en direction. Ceci explique pourquoi la dosimétrie individuelle est essentielle pour cette catégorie de travailleurs. Une dizaine de travailleurs seraient concernés par l'irradiation neutronique pendant le fonctionnement du réacteur. La surveillance dosimétrique des travailleurs devrait s'effectuer mensuellement. L'utilisation des rem-mètres constitue un moyen de surveillance des zones de travail afin de prévenir tout risque de sur-exposition. 
Le dosimètre CR-39 est moins sensible aux effets environnementaux et possède une gamme dynamique de mesure plus étendue que celle du dosimètre à bulles. Ses propriétés dosimétriques sont convenables: pour une incidence normale la réponse en dose équivalente est constante entre $-92 \%$ à $+10 \%$ sur la gamme d'énergies allant de $100 \mathrm{keV}$ à $1 \mathrm{MeV}$, la sensibilité est suffisante, la réponse angulaire est acceptable et la limite de détection est basse. La mauvaise réponse angulaire peut être considérée comme tolérable quand on tient compte des circonstances pratiques d'exposition des travailleurs. En présence des neutrons thermiques et épithermiques, le dosimètre de type CR-39 doit être associé à un convertisseur.

Grâce à leur modeste prix et aux avantages sus-mentionnés, les détecteurs solides de traces de type CR-39 sont les plus appropriés en tant que dosimètres de neutrons pour la surveillance des travailleurs auprès du réacteur TRIGA.

Remerciements. Nous tenons à remercier le professeur F. Vanhavere (SCKCENBelgique) pour son aide bibliographique et ses discussions précieuses.

\section{REFERENCES}

Alberts W.G., Bordy J.M., Chartier J.L., Jahr R., Klein H., Luszik-Bhadra M., Posny F., Schumacher H., Seibert B.R.L. (1996) Neutron dosimetry. Radioprotection 31(1), 37-65.

Al-Jarallah M.I. (1989) Optimization of a single sphere albedo system using helium counters for the measurement of neutron dose equivalent rates and the field calibration of personnel albedo dosimeters, KFK report-N-4632, Karlsruhe.

Bartlett D. (1987) Ageing of and environmental effects on PADC (CR-39), Radiat. Prot. Dosim. $20(1-2), 71-75$.

d'Errico F., Alberts W.G., Matzke M. (1997) Advances in superheated Drop (bubble) detector techniques, Radiat. Prot. Dosim. 70(1-4), 103-108.

Griffith R.V. (1992) Individual neutron monitoring-Needs for the nineties, Radiat. Prot. Dosim. 44(1-4), 259-266.

IAEA (1991) Compendium of neutron spectra and detector responses for radiation protection purposes, Technical Reports Series No. 318. IAEA, Vienna.

IAEA (2002) Compendium of neutron spectra and detector responses for radiation protection purposes: supplement to technical reports series No. 318. Technical reports series No. 403. IAEA, Vienna.

ICRP Publication 60 (1991) Recommendations of the International Commission on Radiological Protection, Ann. ICRP 21(1-3)

JO (1997) Décret 2-97-30 du 28/10/97 pour l'application de la loi 005-71 du 12 octobre 1971 relative à la protection contre les rayonnements ionisànts.

Hadlock D.E., Parkhurst M.A. (1986) Electrochemical development of particle tracks in CR-39 polymer dosimeters, Nucl. Track. 12(1-6), 185-187.

Harvey J.R., Tanner R.J., Alberts W.G., Bartlett D.T., Piesch E.K.A., Schraube H. (1997) The present status of etched track neutron dosimetry in EUROPE and the contribution of CENDOS and EURADOS, Radiat. Prot. Dosim. 70(1-4), 127-132. 
Harvey J.R., Tanner R.J., Alberts W.G., Battlet1 D.T., Piesch E.K.A., Schraube H. (1998) The contribution of EURADOS and CENDOS to etched track neutron dosimetry: The current status in EUROPE, Radiat. Prot. Dosim. 77(4), 267-304.

Kamenopoulou V., Carinou E., Stamatelatos I.E. (2001) Personal neutron dosimetry at a research reactor facility, Radiat. Prot. Dosim. 96(1-3). 197-200.

Lahaye T., Cutarella D., Ménard S., Bolognese-Milsztajn T. (2001) The first operational dosimeter for neutrons which complies with IEC standard 1323, Radiat. Prot. Dosim. 96(1-3), 241-244.

Piesch E. (1982) Albedo neutron dosimetry, Int. J. Apll. Radiat. Isot. 33, 1061-1076.

Serén T.O., Kortesniemi M., Aschan C., Seppälä T., Lampinen J., Auterinen I., Sawolainen S. (1999) A Tale of two Beams : comparison of the radiation fields at the BMRR and FiR 1 epithermal BNCT facilities, Med. Biol. Engineer. Comput. 37(Suppl. 1), 396-397.

Tanner R.J., Thomas D.J., Bartlett D.T., Hager L.G., Horwood N., Taylor G.C. (2002) Effect of the energy dependence of response of personal neutron dosemeters routinely used in the UK on the accuracy of dose estimation. NRPD report W-25, Chilton.

Turek K., Bednar J., Piesch E. (1995) Determination of the neutron angular response using a single etched track detector, Radiat. Prot. Dosim. 59(3), 205-2II

Vanhavere F., d'Ericco F. (2002) Standarisation of superhealed drop and hubble detectors, Radiat. Prot. Dosim. 101(1-4), 283-287.

Vanhavere F., Loos M., Plompen A.J.M., Wattercamps E., Thierensl, H. (1998) A combined use of the BD-PND and BDT bubble detectors in neutron dosimetry, Radiat. Meas. 29(5), 573-577.

Vanhavere F., Loos M., Thierens1 H. (1999) the life span of the BD-PND bubble detector, Radiat. Prot. Dosim. 85(1-4), 27-30.

Vareille J.C., Barelaud B., Barthe J., Bordy J.M., Curzio G.. D'Ericco F., Decossas J.L. (1997) Advances detectors for active neutron dosemeters, Radiat. Prot. Dosin. 70 (1-4), 79-82.

Wernli C., Barthe J., Dietze G. (1996) Individual dosimetry, Radioprotection 31(1), 17-35. 